\title{
Primary Extraskeletal Osteosarcoma Arising from the Optic Nerve: A Rare Case Report
}

\author{
Bifica Lyngdoh $^{a} \quad$ Jaya Mishra $^{a} \quad$ Biswajit Dey $^{a} \quad$ Caleb Harris $^{\mathrm{b}} \quad$ Arindom Kakatic $^{\mathrm{c}}$ \\ Zachariah Chowdhury $^{\mathrm{a}}$ Rintu Marak ${ }^{\mathrm{b}}$ Evarisalin Marbaniang ${ }^{\mathrm{a}}$ \\ aDepartment of Pathology, North Eastern Indira Gandhi Regional Institute of Health and Medical Sciences, \\ Shillong, India; b Department of Oncology, North Eastern Indira Gandhi Regional Institute of Health and Medical \\ Sciences, Shillong, India; ' Department of Neurosurgery, North Eastern Indira Gandhi Regional Institute of \\ Health and Medical Sciences, Shillong, India
}

\section{Established Facts}

- Orbital location of extraskeletal osteosarcoma is extremely rare with only 4 cases reported so far in the English literature.

- All 4 reported patients of primary orbital extraskeletal osteosarcoma were males.

\section{Novel Insights}

- The present case is the first case of primary orbital extraskeletal osteosarcoma occurring in a female.

- Alterations of p53 and RB1 genes in extraskeletal osteosarcoma are similar to that of skeletal osteosarcoma.

\section{Keywords}

Osteoid · Orbit · Vimentin

\section{Abstract}

Orbital location of extraskeletal osteosarcoma is extremely rare with only 4 cases reported so far in the English literature. We present the case of a 32-year-old female who presented with proptosis and complete loss of vision of the left eye. Contrast-enhanced computed tomography scan showed a densely calcified lobulated lesion in the left optic nerve showing strong enhancement. A left fronto-occipito-zygomatic osteotomy was conducted and a greyish brown tumor was identified. Histopathological and immunohistochemical examination of the curetted material revealed it to be extraskeletal osteosarcoma. A left partial maxillectomy with ethmoidectomy and left orbital exenteration was done and the patient was advised chemotherapy with radiotherapy.

(c) 2018 S. Karger AG, Basel

\section{KARGER}

E-Mail karger@karger.com

www.karger.com/oop
(C) 2018 S. Karger AG, Basel Dr. Jaya Mishra

Department of Pathology

NEIGRIHMS

Shillong 793018 (India)

E-Mail drjayamshillong@gmail.com 
Fig. 1. a Patient showing proptosis of the left eye. b Computed tomography scan showing a well-circumscribed enhancing tumor in the retro-orbital region. c MRI showing a fusiform mass arising from the postero-infero-medial aspect of the left optic nerve. d Curetted tumor tissue.
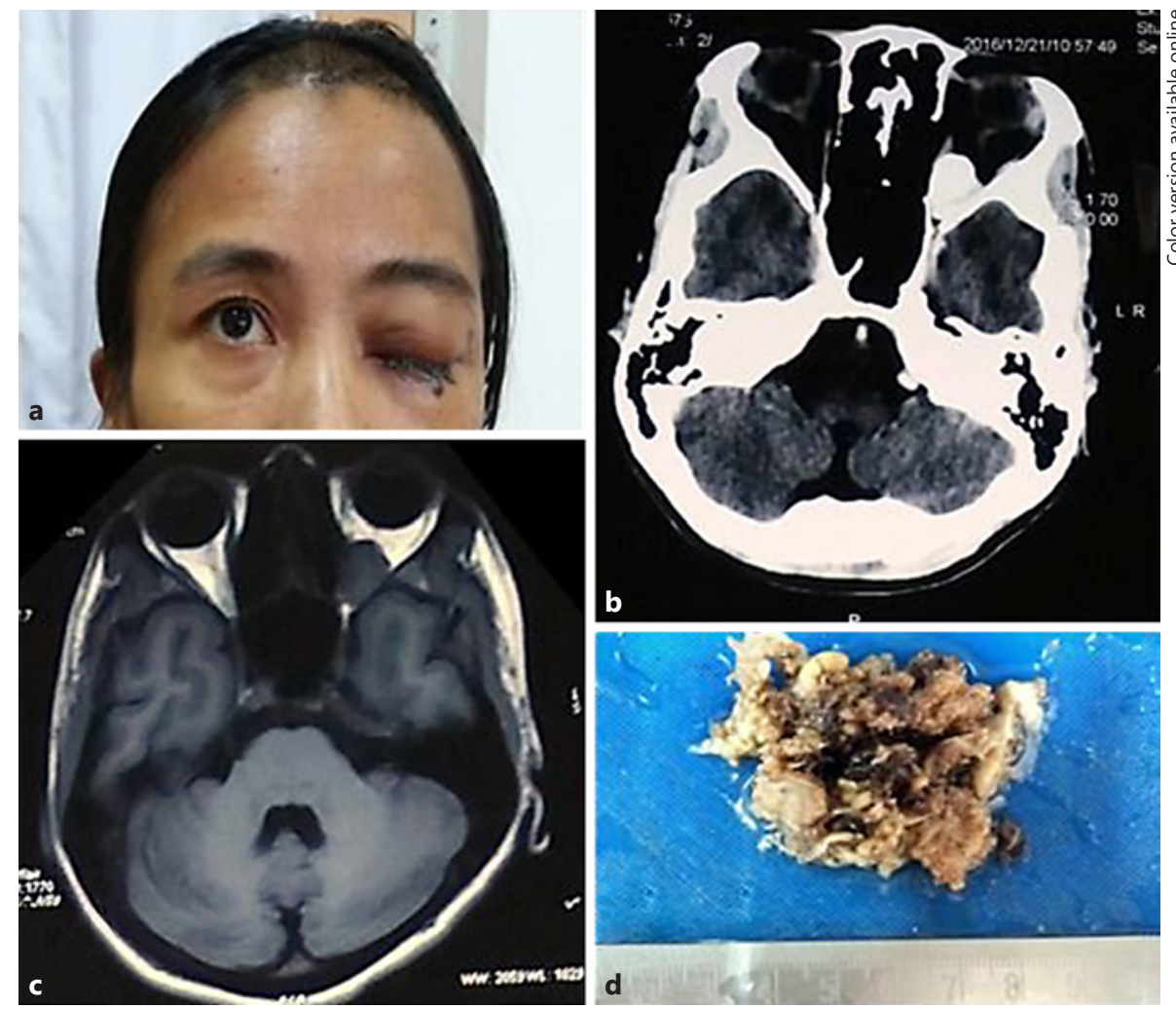

\section{Introduction}

Osteosarcoma is the most common primary malignant bone tumor excluding the hematopoietic malignancies [1]. It is usually located in the metaphyseal region of the long bones. It rarely occurs in the head and neck region comprising of $6-13 \%$ as primary tumor in this region [1]. Extraskeletal osteosarcoma (EOS) accounts for $2-4 \%$ of all osteosarcomas and is extremely rare. Only 4 cases of EOS in the orbital region have been reported in the English literature so far [2-5]. We report a case of primary EOS of the left optic nerve in a 32-year-old female who presented with proptosis and complete loss of vision of the left eye.

\section{Case Report}

A 32-year-old female presented with increasing proptosis of the left eye for the last 6 months (Fig. 1a). She gave a history of blurring vision of the left eye for 6 months with complete loss of vision of the same eye for 1 month. There was no history of orbital trauma. The patient had no history of previous malignancies or irradiation. Examination of the left eye showed features of compressive optic neuropathy and cilioretinal artery ischemia. Right eye examination was normal except for mild disc edema.
Contrast-enhanced computed tomography scan showed a densely calcified lobulated lesion in the left optic nerve showing strong enhancement (Fig. 1b). Computed tomography of the brain and bony calvarium showed normal study.

Magnetic resonance imaging (MRI) scan of her brain showed a fusiform mass measuring $23 \times 15 \times 15 \mathrm{~cm}$ arising from the postero-infero-medial aspect of the left optic nerve (Fig. 1c). The lesion was isointense on $\mathrm{T} 1$ and isointense to mildly hyperintense on T2 sequences. Dense nodular calcification was seen in the lesion. The right optic nerve and optic chiasma was normal. No significant abnormality was seen in the brain. Radiological differential diagnoses were left optic nerve sheath tumor and optic nerve meningioma.

A left fronto-occipito-zygomatic osteotomy was conducted and a greyish brown tumor was identified. It was curetted and sent for histopathological examination. Grossly, there were multiple friable grey white tissues with attached part of left optic nerve (Fig. 1d).

Histopathological examination showed a diffusely infiltrating cellular mass arranged in lobules and sheets (Fig. 2a, b). The tumor cells were highly pleomorphic with eccentric nucleus and dark eosinophilic cytoplasm and prominent nucleoli. Occasional tumor giant cells were also seen. The tumors had areas of actively synthesizing osteoid (Fig. 2c, d). Mitotic count was brisk. On immunohistochemistry (IHC), vimentin was strongly positive in the tumor cells (Fig. 3a). Epithelial membrane antigen (EMA), glial fibrillary acidic protein (GFAP), S100, and myogenin were negative in the tumor cells (Fig. 3b). Based on histomorphology and IHC, a diag- 
Fig. 2. a, b Histopathology showing a diffusely infiltrating cellular mass arranged in lobules and sheets (H\&E, 40×). c, d Tumor showing highly pleomorphic tumor cells with eccentric nucleus and dark eosinophilic cytoplasm and prominent nucleoli with intervening malignant osteoid formation (H\&E, 400×).

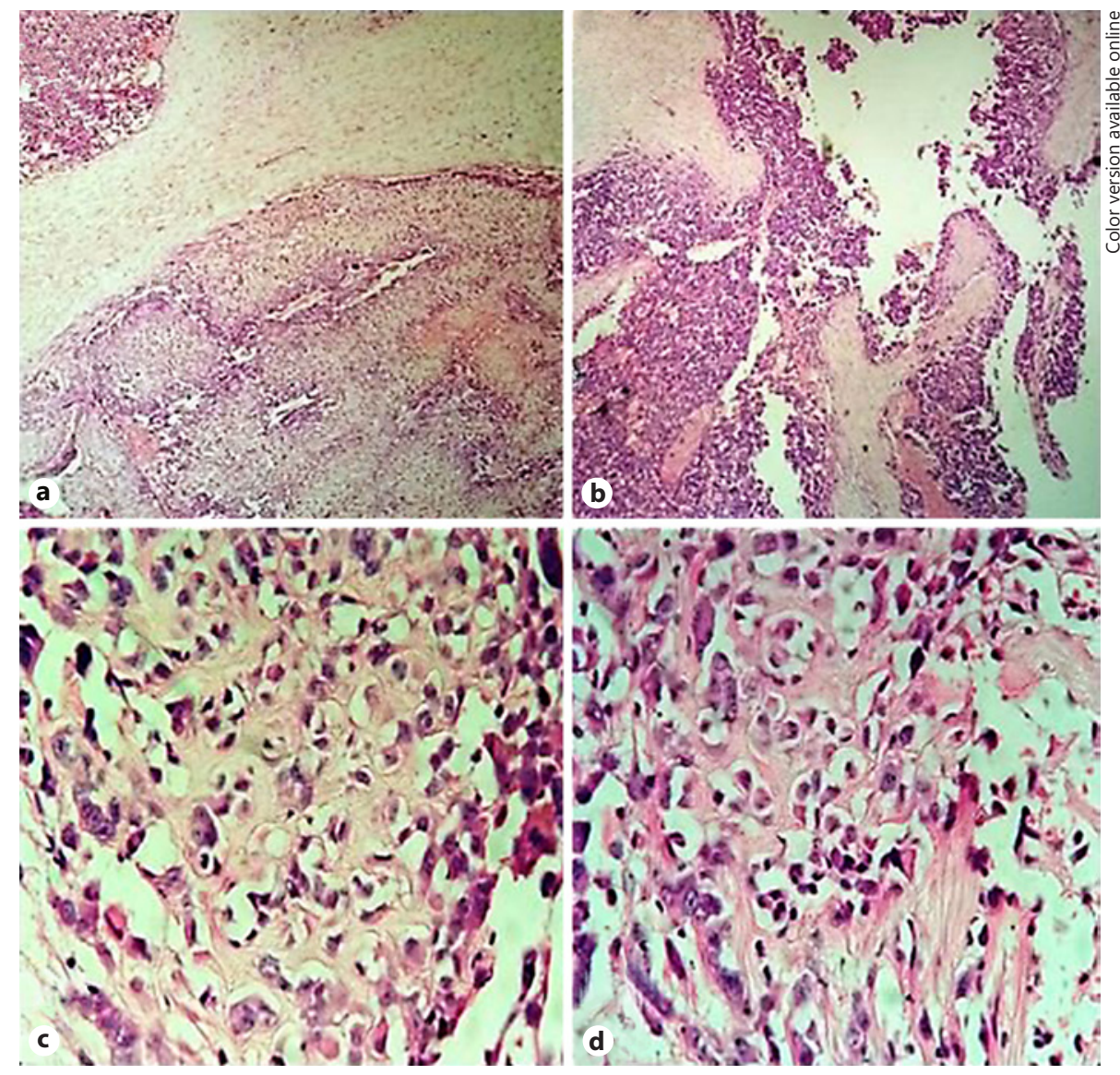

nosis of osteosarcoma was made. A bone scan was done to rule out metastasis. Bone scan revealed no other lesions in the body ruling out metastasis to the left optic nerve. So a diagnosis of primary EOS of the left optic nerve was made.

Assessments of p53 and RB1 were done immunohistochemically. Overexpression of $\mathrm{p} 53$ and reduced expression of $R B 1$ were observed in the tumor cells (Fig. 3c, d).

A left partial maxillectomy with ethmoidectomy and left orbital exenteration was done. The patient was referred to the oncology section for chemotherapy and radiotherapy. However the patient never turned up for further management and was lost to follow-up.

\section{Discussion}

In the vast majority of osteosarcomas arising within bone, the osteosarcoma usually starts within bone, even though some of them have a prominent extraosseous component [6]. They usually arise from the metaphysis of the long bones and are high-grade malignant tumors [6]. Most osteosarcomas occur in adolescents and young adults and are rare in those younger than 5 years or old- er than 50 years unless there is a predisposing condition [6].

Osteosarcoma in the head and neck region is relatively rare with mandible and maxilla being the common sites [7]. Orbital region is an extremely rare location for osteosarcoma [7]. The clinical manifestations of head and neck osteosarcomas depend on the location of the tumor. Mandibular lesions present with focal pain and swelling, whereas osteosarcomas involving the maxilla, paranasal sinuses, and orbit are less likely to be painful [7]. In the present case, the patient presented with ocular symptoms of proptosis and blurring of vision followed by loss of vision.

EOS is extremely rare and accounts for $2-4 \%$ of all osteosarcomas. Unlike skeletal osteosarcoma patients who present in the fifth and sixth decades of life, patients with EOS usually present in the fourth and fifth decades of life [6]. The commonly involved regions are the extremities and girdles. Only 4 cases of EOS in the orbital region have been reported in the English literature (Table 1) [2-5]. EOS involving the face, breast, abdominal wall, soft tis- 
Fig. 3. a Vimentin positivity in the tumor cells (IHC, 400×). b GFAP negativity in the tumor cells (IHC, 40×). c p53 positivity in the tumor cells (IHC, 400×). d Downregulation of Rb1 in the tumor cells (IHC, 40x).

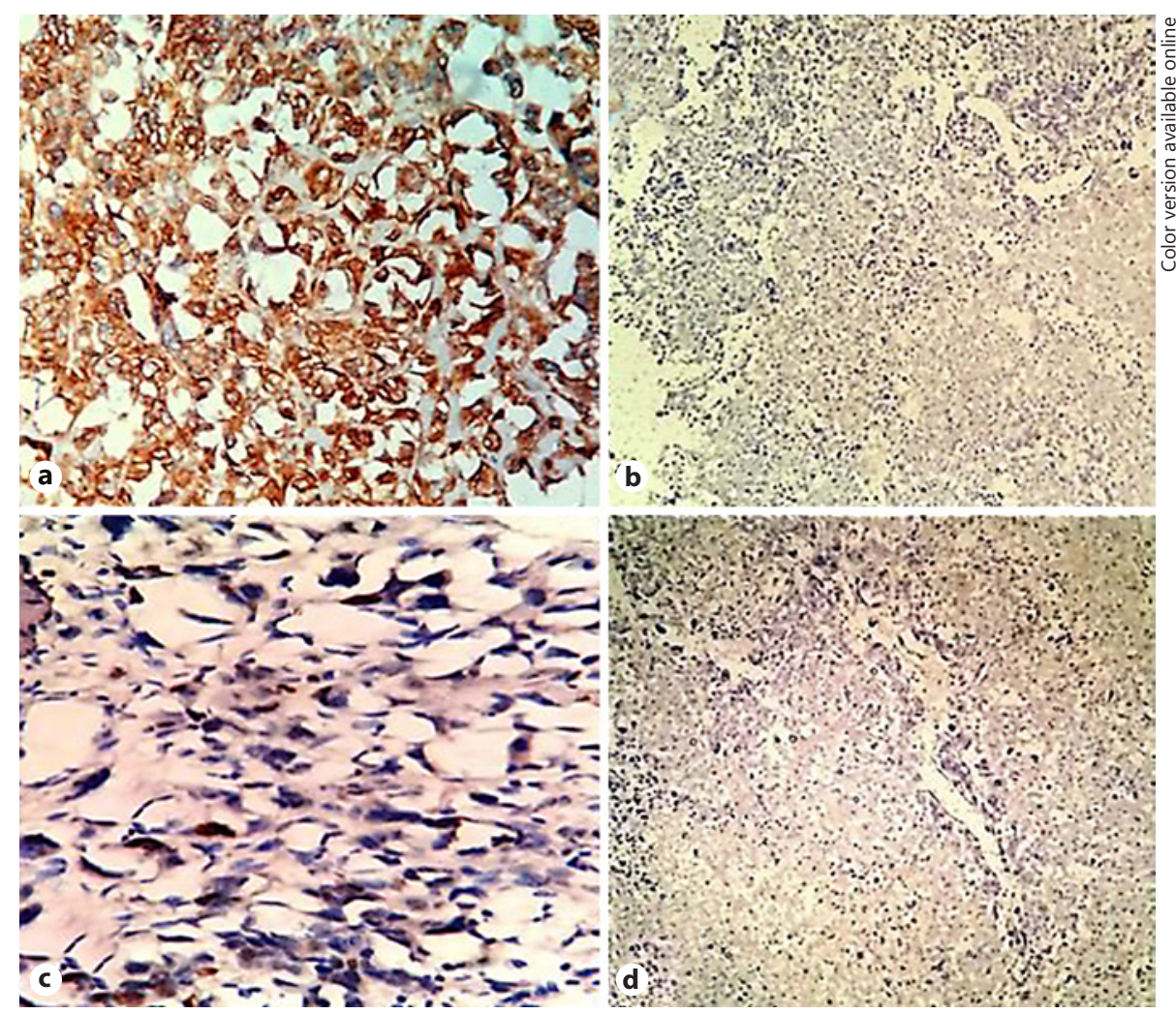

Table 1. Cases of extraskeletal osteosarcoma in the orbital region reported in the literature

\begin{tabular}{|c|c|c|c|c|c|c|}
\hline $\begin{array}{l}\text { Ref. } \\
\text { No. }\end{array}$ & Author & Year & Age, years & Sex & Primary malignancies & $\begin{array}{l}\text { Prior history } \\
\text { irradiation }\end{array}$ \\
\hline [2] & Kauffman and Stout & 1963 & 11 & Male & Retinoblastoma & Present \\
\hline$[4]$ & Fan et al. & 2011 & 78 & Male & Basal cell carcinoma & Present \\
\hline [5] & De Maeyer et al. & 2016 & 32 & Male & Absent & Absent \\
\hline
\end{tabular}

sues of the back and retroperitoneum, and site of a vaccination scar have also been reported [8-10]. All 4 reported patients of primary orbital EOS were males. The present case is the first case of primary orbital EOS occurring in a female.

Radiotherapy is a known predisposing factor. Sordillo et al. [8] found that $10 \%$ of the patients had previous irradiation and $13 \%$ of the patients had a history of trauma related to the site of EOS. In the present case, the patient had no history of trauma or radiotherapy.

While MRI offered excellent resolution of the tumor extent, it is unable to reveal any information on the matrix calcification [11]. In this regard, computed tomogra-

Primary Extraskeletal Osteosarcoma Arising from the Optic Nerve phy scan enables a better characterization of the osseous matrix and cortical destruction [11].

Histopathologically, osteosarcoma has proliferating malignant cells that produce a characteristic osteoid matrix. The matrix may be in the form of unmineralized osteoid or may show mineralized trabeculae. The histopathological differential diagnoses of primary orbital EOS include hyperostotic meningioma, malignant peripheral nerve sheath tumor, glioblastoma, gliosarcoma, and rhabdomyosarcoma. So a panel of IHC markers is required to arrive at the final diagnosis. In the present case, the tumor cells were positive for vimentin. The differential diagnoses of hyperostotic meningioma (negative ex-

Ocul Oncol Pathol 2018;4:304-308 
pression of EMA), malignant peripheral nerve sheath tumor (negative expression of S100), glioblastoma and gliosarcoma (negative expression of GFAP), and rhabdomyosarcoma (negative expression of myogenin) were ruled out based on IHC findings.

The most common genetic changes associated with osteosarcoma are the alterations of the p53 tumor suppressor gene and retinoblastoma gene $R B 1$ [5]. In the present case, overexpression of $\mathrm{p} 53$ and reduced expression of $R B 1$ were seen suggesting alterations of the p53 and $R B 1$ gene.

Primary EOS is aggressive in nature. The standard treatment consists of radical surgery and polychemotherapy [12]. No data from randomized studies concerning the type of chemotherapeutic combinations are available due to the rare nature of this disease. In a small retrospec- tive study by Goldstein-Jackson et al. [12], EOS were treated as conventional osteosarcoma, and a combination of multi-agent chemotherapy with surgery was given and surprisingly had a good survival rate.

\section{Statement of Ethics}

This is a case report and our institute does not require Institute Ethics Committee approval for case reports. Informed consent has been taken from the patient for publication of this case report.

\section{Disclosure Statement}

The authors declare that no conflicts of interest and no sponsorship or funding was received for this research.

\section{References}

1 Ha PK: Osteosarcoma of the head and neck. A review of the John Hopkins experience. Laryngoscope 1999;109:964-969.

2 Kauffman SL, Stout AP: Extraskeletal osteogenic sarcomas and chondrosarcomas in children. Cancer 1963;16:432-439.

3 Jacob R, Abraham E, Jyothirmayi R, Nair MK Extraskeletal osteosarcoma of the orbit. Sarcoma 1998;2:121-124.

4 Fan JC, Lamont DL, Greenbaum AR, Ng SG: Primary orbital extraskeletal osteosarcoma. Orbit 2011;30:297-299.
5 De Maeyer VMDS, Kestelyn PAFA, Shah AD, Van Den Broecke CM, Denys HGN, Decock CE: Extraskeletal osteosarcoma of the orbit: a clinicopathologic case report and review of literature. Indian J Ophthalmol 2016;64:687689.

6 Ottaviani G, Jaffe N: The etiology of osteosarcoma. Cancer Treat Res 2009;152:15-32.

7 Laskar S, Basu A, Muckaden MA, et al: Osteosarcoma of the head and neck region: lessons learned from a single-institution experience of 50 patients. Head Neck 2008;30:10201026.

8 Sordillo P, Hajdu SI, Magill GB, Golbey RB: Extra skeletal osteosarcoma: a review of 48 cases. Cancer 1983;51:727-734.
9 Rao U, Cheng A, Didolkar MS: Extraosseous osteogenic sarcoma: clinico-pathological study of eight cases and review of literature. Cancer 1978;41:1488-1496.

10 Fine G, Stout AP: Osteogenic sarcoma of the extraskeletal soft tissues. Cancer 1956;9: 1027-1043.

11 Wei-Min Tsai, Yung-Cheng Wang, Jan-Wen $\mathrm{Ku}$, Chi-Jen Chen, Jing-Shan Huang: Orbital osteosarcoma masqueraded as hyperostotic meningioma. J Radiol Sci 2011;36:241-246.

12 Goldstein-Jackson SY, Gosheger G, Delling $\mathrm{G}$, et al: Extraskeletal osteosarcoma has a favourable prognosis when treated like conventional osteosarcoma. J Cancer Res Oncol 2005; 13:520-526. 